\title{
Metallomics
}

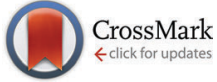

Cite this: Metallomics, 2016, 8,774

Received 3rd March 2016 Accepted 29th April 2016

DOI: 10.1039/c6mt00051g

www.rsc.org/metallomics

\section{Exploring the urinary selenometabolome following a multi-phase selenite administration regimen in humans $\dagger$}

\begin{abstract}
Bassam Lajin, Doris Kuehnelt and Kevin A. Francesconi*
To gain more insight into the human metabolism of the essential trace element selenium, we investigate the response of the urinary selenium metabolites to changing selenium intake by applying a stepwise selenium administration regimen based on repeated dosaging. Sodium selenite was administered orally to healthy volunteers at an incrementally increasing dosage. The supplementation regimen extended over 20 days for each volunteer, and daily morning urine samples were collected prior to, during, and following the supplementation phases. A total of 160 urine samples were analyzed for total urinary selenium and a panel of selenometabolites by using ICPMS and HPLC/ICPMS. Selenosugar 1 gave the strongest response followed by TMSe and then selenosugar 3. Se-methylselenoneine excretion was not stimulated by increased selenium intake, suggesting that it is not in equilibrium with selenium body pools. Selenate was detected in all urine samples; it showed a clear and consistent response to supplementation and an abrupt return to baseline levels upon cessation of supplementation, indicating that it arose from the oxidation of the administered selenite rather than from the oxidation of endogenous hydrogen selenide. The gap between total urinary selenium and the sum of Se species markedly increased in response to selenium administration, which highlights the presence of unknown $\mathrm{Se}$ species that respond to selenite supplementation. The characterization of these unknown species and their possible biological activities might be essential before considering selenium supplementation in clinical trials. We discuss the implications of the responses of the selenium metabolites and their inter-relationships for selenium metabolism.
\end{abstract}

The biochemical pathways of the biotransformation of the essential trace element selenium are poorly understood. By applying a stepwise multi-phase regimen for selenium administration in human volunteers including pre-supplementation and post-supplementation analyses, the present work contributes to a better understanding of the relationship between the different components of the urinary selenometabolome, their relationship with the central metabolite hydrogen selenide, and their positioning on the selenium metabolic network.

\section{Introduction}

Despite selenium's low abundance in the earth's crust, the catalytic advantages of replacing sulfur by selenium in selenoproteins warranted the evolution of a highly complicated mechanism for the co-translational insertion of selenocysteine, the 21 st amino acid, in proteins in response to a stop codon. ${ }^{1}$

Institute of Chemistry-Analytical Chemistry, NAWI Graz, University of Graz, Universitaetsplatz 1, 8010 Graz, Austria. E-mail: kevin.francesconi@uni-graz.at $\dagger$ Electronic supplementary information (ESI) available. See DOI: 10.1039/ c6mt00051g
On the other hand, the high specificity of these advantages is reflected by the limited number of known selenoproteins. ${ }^{2}$

Although the essentiality of selenium was first recognized over 60 years ago, ${ }^{3}$ only little is known about the biotransformation pathways and enzymatic systems involved in the metabolism of selenium (Fig. 1). The most predominant form of selenium in our diet, selenomethioneine, is either converted into selenocysteine via the transsulfuration pathway ${ }^{4}$ or nonspecifically incorporated into body proteins in place of methionine. ${ }^{5}$ Selenocysteine, originating from the diet or from the catabolism of selenoproteins, is converted into hydrogen selenide by a selenium-specific enzyme, selenocysteine lyase. ${ }^{6}$ The inorganic selenium form selenite is converted into hydrogen selenide 


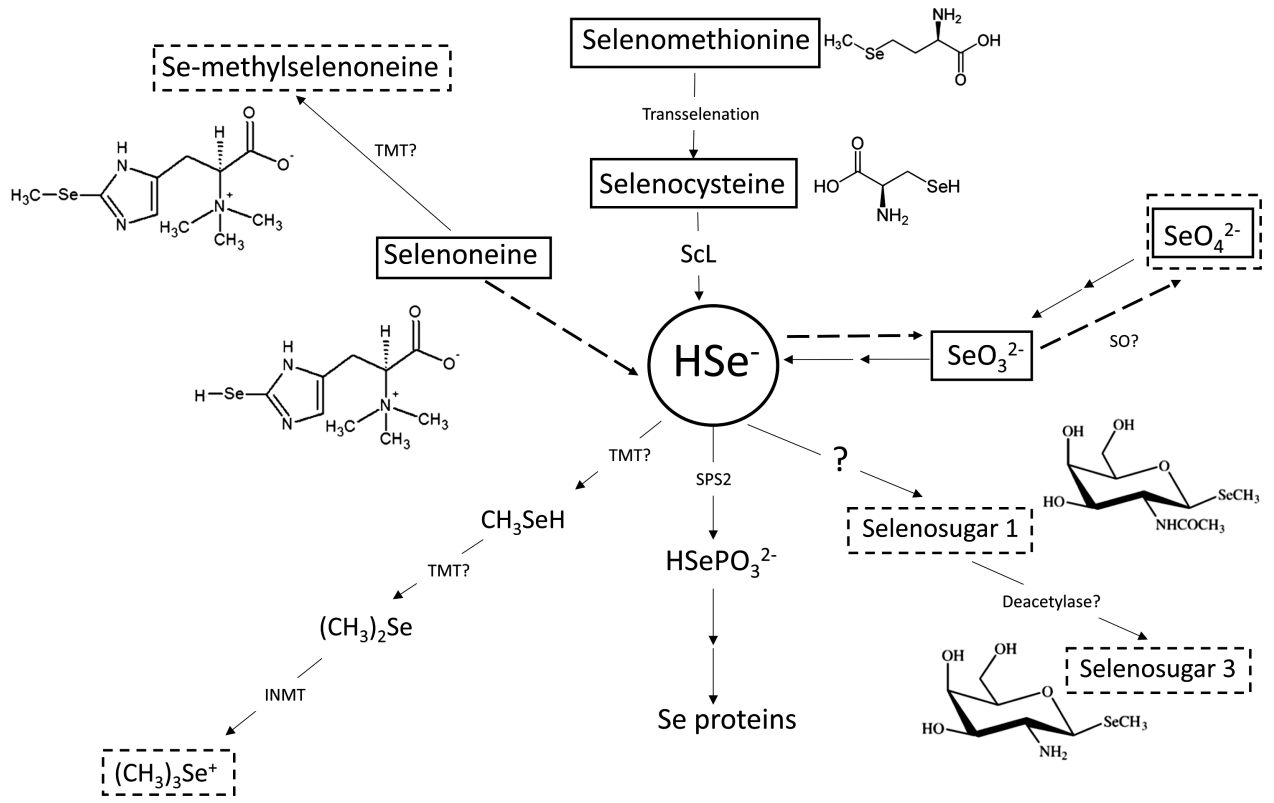

Fig. 1 The selenium metabolic network centered on hydrogen selenide. Selenium species in dashed boxes are urinary metabolites. Selenium species in solid boxes are dietary forms. Dashed arrows indicate an uncertain pathway. Question marks indicate unknown or proposed enzymes. ScL, selenocysteine lyase; SPS2, selenophosphate synthase 2; SO, sulfite oxidase; INMT, indolethylamine N-methyltransferase; TMT, thiol S-methyltransferase.

through the glutathione ${ }^{7}$ and thioredoxin systems. ${ }^{8,9}$ Hydrogen selenide is then used for the synthesis of the active selenium donor for selenoprotein synthesis, selenophosphate, by a selenophosphate synthase, ${ }^{10}$ while the excess is metabolized and excreted in the form of selenosugars and trimethylselenonium ions (TMSe).$^{11}$ Very little is known about other possible fates of the central selenium species hydrogen selenide, and a large gap between the sum of identified urinary selenium species and total urinary selenium has been consistently reported. ${ }^{12,13}$

Numerous studies explored the urinary excretion of selenium supplemented in various chemical forms, but based on different experimental designs employing single dosage administration. ${ }^{12,14-16}$

The aim of the present study is to investigate the urinary excretion of selenium metabolites in several volunteers following a regimen of a gradually increasing dosage of selenium supplementation to gain more insight into the human metabolism of selenium.

\section{Materials and methods}

\section{Selenium ingestion and sample collection}

The study cohort consisted of 8 non-smoking volunteers ( 4 females and 4 males) not taking multivitamin supplements. Basic information about the volunteers is summarized here as range, mean \pm SD. Age: $26-61$ years, $41 \pm 13$; BMI: 22-29 $\mathrm{kg} \mathrm{m}^{-2}, 25 \pm 2$; metabolic body weight: $22-30 \mathrm{~kg}^{0.75}$, $25 \pm 3$. Volunteers gave informed consent to participate in the study, and all procedures were in accordance with the Declaration of Helsinki.
Each volunteer was asked to collect a morning urine sample after waking up (first urine pass of the day) over 20 consecutive days while taking selenium supplements at bedtime according to the regimen illustrated in Fig. 2. The administered selenium was in the form of commercial tablets containing $100 \mu \mathrm{g}$ Se in the chemical form sodium selenite (Selamin ${ }^{\circledR}$, Erwo Pharma $\mathrm{GmbH}$, Pfaffstaetten, Austria). The volunteers were asked to record general dietary information to account for selenium-rich diets. Urine was collected in Corning ${ }^{\mathbb{R}}$ polypropylene $300 \mathrm{~mL}$ sample collection containers (Corning, NY, USA). Three subsamples,

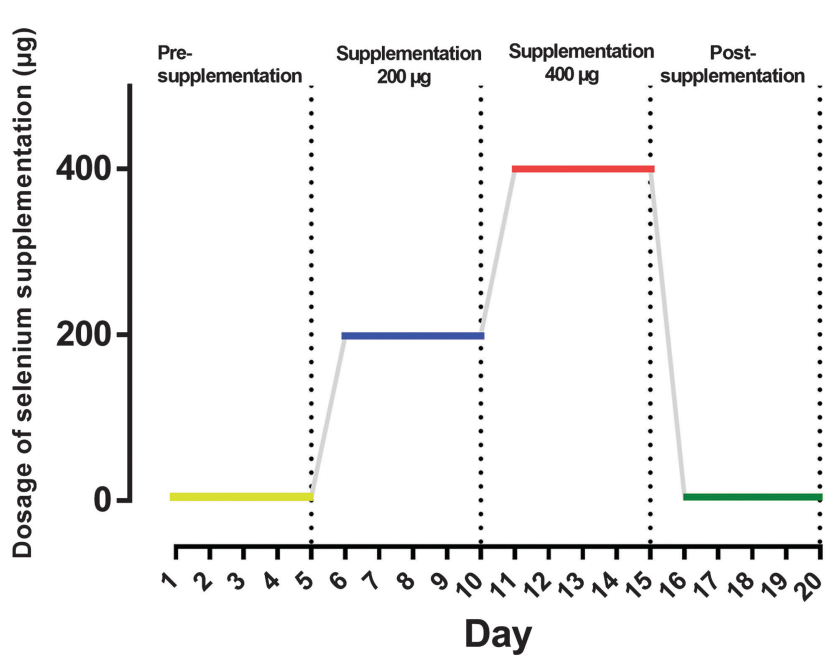

Fig. 2 The supplementation regimen applied in the present study. Day $1-5$, no supplementation; day $6-10$, supplementation with $200 \mu \mathrm{g}$ selenium; day $11-15$, supplementation with $400 \mu \mathrm{g}$ selenium; day $16-20$, no supplementation. 
each about $5 \mathrm{~mL}$, were taken from each donated urine sample and stored at $-80{ }^{\circ} \mathrm{C}$ until analysis.

\section{Determination of total selenium in urine by ICPMS}

Microwave-assisted acid digestion was used to mineralize the urine samples ( $500 \mu \mathrm{L}$ portions) with nitric acid employing the conditions previously described. ${ }^{16}$ The resulting digest solution was made up to $10 \mathrm{~mL}$ with water $(18.2 \mathrm{M} \Omega \mathrm{cm})$ and the total selenium content was determined by using an inductively coupled plasma mass spectrometer (ICPMS, Agilent 7900, Agilent Technologies, Waldbronn) applying the conditions previously described. ${ }^{16}$ In brief, we operated in the octopole reaction cell mode with hydrogen as the reaction gas. An optional gas containing $1 \% \mathrm{CO}_{2}$ in argon was used ${ }^{17}$ to provide a four-fold increase in sensitivity for selenium provided by the presence of carbon in the plasma. ${ }^{18}{ }^{73} \mathrm{Ge}$ was used as the internal standard. The limit of detection for the total selenium determinations $\left({ }^{78} \mathrm{Se}\right.$ ) was $2 \mathrm{ng} \mathrm{L}{ }^{-1}$ (based on blank $+3 \mathrm{SD}_{\text {blank }}$ ). The certified reference material NIES CRM 18 Human Urine (National Institute of Environmental Studies, Tsukuba, Japan) was used to validate our total selenium measurements (certified Se concentration: $59 \pm 5 \mu \mathrm{g} \mathrm{L^{-1 }}$; found Se concentration: $\left.60 \pm 2 \mu \mathrm{g} \mathrm{L}^{-1}, n=3\right)$. Se concentrations in urine were normalised according to specific gravity determined on a Leica TS 400 total solids refractometer (Leica Microsystems, Buffalo, NY, USA) using the following equation: $C_{\text {normalized }}=$ $C_{\text {measured }}\left(\mathrm{SG}_{\text {average }}-1\right) /\left(\mathrm{SG}_{\text {sample }}-1\right) .{ }^{19}$

\section{Determination of selenium species in urine by HPLC/ICPMS}

Three sets of chromatographic conditions were used for the determination of the selenium metabolites applied as previously described ${ }^{16}$ with slight modifications. Reversed-phase chromatography was employed to determine selenosugar 1 and Se-methylselenoneine. A Waters Atlantis C18 column (4.6 $\times$ $150 \mathrm{~mm}$; Waters Corporation, Milford, MA, USA) was used with a mobile phase of $20 \mathrm{mM}$ ammonium formate adjusted with formic acid to $\mathrm{pH}=3.0$ and containing $3 \%$ methanol, at a flow rate of $1.0 \mathrm{~mL} \mathrm{~min}^{-1}$ and a column temperature of $30{ }^{\circ} \mathrm{C}$. A Hamilton PRP-X200 strong cation-exchange column $(4.1 \times$ $250 \mathrm{~mm}$; Hamilton, Reno, NV, USA) was used to separate selenosugar 3 and trimethylselenonium (TMSe), employing $20 \mathrm{mM}$ ammonium formate adjusted with formic acid to $\mathrm{pH}=3.0$ as the mobile phase, with a flow rate of $1.4 \mathrm{~mL} \mathrm{~min}^{-1}$ at a column temperature of $50{ }^{\circ} \mathrm{C}$. For the separation and quantification of selenite and selenate, anion-exchange chromatography was performed on a PRP-X100 column $(4.1 \times 150 \mathrm{~mm}$; Hamilton, Reno, NV, USA) with a mobile phase of $20 \mathrm{mM}$ ammonium malonate adjusted to $\mathrm{pH}=9.5$ with ammonia, at a

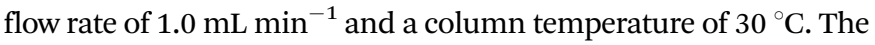
injection volume was $20 \mu \mathrm{L}$ for all chromatographic conditions. For both cation-exchange and anion-exchange chromatography, which did not use methanol in the mobile phase, a plasma optional gas of $1 \% \mathrm{CO}_{2}$ in argon was used for the ICPMS detector ${ }^{17}$ to provide a 4 -fold increase in sensitivity. ${ }^{18}$

The urine samples were filtered using nylon syringe filters (pore size $0.2 \mu \mathrm{m}$, VWR International, USA) prior to injection onto the column. ICPMS was used for the selective detection of selenium-containing species at $m / z$ 78. The external standards used for the quantification of selenium metabolites were purchased (selenite and selenate) or synthesized in-house (selenosugar 1, methyl-2-acetamido-2-deoxy-1-seleno- $\beta$-D-galactopyranoside; selenosugar 3, methyl-2-amino-2-deoxy-1-seleno- $\beta$-D-galactopyranoside; and TMSe), as previously described..$^{12,20-22}$ Because a standard of Se-methylselenoneine was not available, and since the ICPMS is an element-dependent detector, this metabolite was quantified based on the TMSe standard. The presence of Se-methylselenoneine in urine was identified by spiking with Se-methylselenoneine purified from the tuna extract as previously described. ${ }^{23}$

The limit of detection (based on $3 \times \mathrm{SE}_{y}$, standard error of the $y$-intercept of a calibration curve recorded from 0.1 to $5.0 \mu \mathrm{g} \mathrm{Se} \mathrm{L}^{-1}$ ) for the selenium species with the chromato-

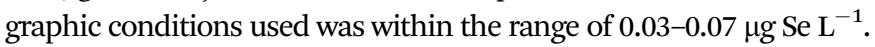
The limit of quantification $\left(10 \times \mathrm{SE}_{y}\right)$ for the selenium species was within the range of $0.1-0.2 \mu \mathrm{g}$ Se $\mathrm{L}^{-1}$. The concentrations are reported as selenium mass per volume $\left(\mu \mathrm{g}\right.$ Se $\left.\mathrm{L}^{-1}\right)$ after normalizing the data according to specific gravity as described above.

\section{Analysis of the selenium supplement}

We checked the commercial selenium tablets before administration to volunteers both quantitatively and qualitatively. Nine tablets were randomly selected, and individually weighed and ground to a powder. Portions of $50 \mathrm{mg}$ of the powder of each tablet were suspended in $30 \mathrm{~mL}$ of water in $50 \mathrm{~mL}$ polypropylene tubes and extracted by shaking the mixture overnight at room temperature; the extract was then measured for Se species by anion-exchange HPLC (see chromatographic conditions above). For total Se content portions of $50 \mathrm{mg}$ of the powder of each tablet were digested using microwave-assisted acid digestion and the digest was analyzed for total Se as described above. The tablets $(n=9)$ were found to contain $102 \pm 10 \mu \mathrm{g}$ total selenium and $106 \pm 8 \mu \mathrm{g}$ Se as selenite.

\section{Results and discussion}

\section{A general overview}

All volunteers had comparable dietary habits except for volunteers A and D who had higher fish consumption (1 per week versus 1 per month for the other volunteers). The total urinary selenium excretion followed the expected pattern based on the supplementation regimen applied (Fig. 3).

Our chromatographic systems detected selenosugar 1, selenosugar 3, Se-methylselenoneine, TMSe, selenite, and selenate in the urine samples of our volunteers (examples of typical chromatograms are provided in the ESI, $\dagger$ Fig. S1). Table 1 shows an overview of the urinary levels of the selenium metabolites studied. The multi-phase stepwise experimental design was utilized to investigate the metabolic network of selenium, centered on hydrogen selenide (Fig. 1). First, monitoring the incremental increase in the urinary concentration of each 

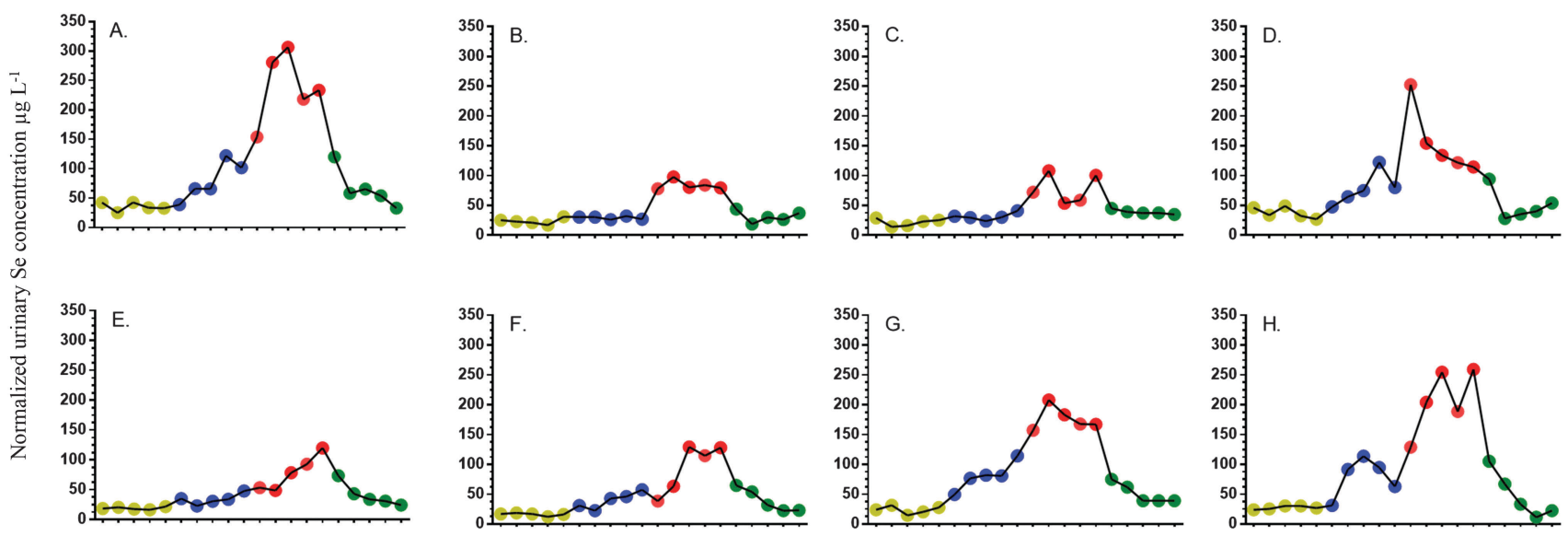

Fig. 3 Total urinary selenium excretion over 20 days in the 8 volunteers (A-H). The study phases are highlighted by different colors (see Fig. 2).

Table 1 An overview of the levels of selenium metabolites quantified in the present study expressed as range (mean). Concentrations are reported in $\mu \mathrm{g} \mathrm{Se} \mathrm{L}^{-1}$. TMSe levels were reported for the TMSe producers. For TMSe nonproducers the levels were within the range $<\mathrm{LOD}-0.2 \mu \mathrm{g}$ Se $\mathrm{L}^{-1}$

\begin{tabular}{|c|c|c|c|c|c|}
\hline & LOD/LOQ & Pre-supplementation & $\begin{array}{l}\text { Supplementation } \\
\text { with } 200 \mu \mathrm{g}\end{array}$ & $\begin{array}{l}\text { Supplementation } \\
\text { with } 400 \mu \mathrm{g}\end{array}$ & Post-supplementation \\
\hline Selenosugar 1 & $0.03 / 0.1$ & $0.9-24.4(3.7)$ & $0.7-75.6(22.5)$ & $11.7-183(80.8)$ & $2.3-56.6(15.6)$ \\
\hline Selenosugar 3 & $0.07 / 0.23$ & $0.8-4.1(2.3)$ & $1.0-9.1(3.9)$ & $2.7-16.7(9.0)$ & $1.4-11.6(4.9)$ \\
\hline Se-methylselenoneine & $0.05 / 0.16$ & $0.2-3.0(0.9)$ & $0.3-2.3(0.7)$ & $0.2-3.9(1.0)$ & $0.2-3.0(0.9)$ \\
\hline Selenate & $0.06 / 0.20$ & $>$ LOD $-0.3(0.2)$ & $0.4-4.3(1.3)$ & $0.6-9.1(2.5)$ & $>$ LOD $-0.4(0.2)$ \\
\hline Selenite & $0.06 / 0.20$ & $<\mathrm{LOQ}$ & $<\mathrm{LOQ}$ & $<\mathrm{LOQ}$ & $<\mathrm{LOQ}$ \\
\hline
\end{tabular}

selenium metabolite starting from the pre-supplementation phase moving to the first $(200 \mu \mathrm{g})$ then the second $(400 \mu \mathrm{g})$ supplementation phase allowed us to order the selenium metabolites in terms of their response to selenium intake. Second, monitoring the urinary levels of the selenium metabolites after cessation of supplementation (post-supplementation phase) enabled us to examine the relationship of these metabolites with the central species hydrogen selenide which reflects selenium body pools. Fig. 4 shows the response of the selenium species to selenium supplementation in all volunteers, reported as the normalized concentration and the percentage of the total urinary selenium. Bearing in mind the above aspects of the applied experimental design, we discuss each detected component of the urinary selenometabolome individually in the following subsections.

\section{Selenosugars}

Selenosugar 1 showed a much stronger response to selenite supplementation (Fig. 4A) than selenosugar 3, which is in agreement with the previously published data. ${ }^{12,14}$ The selenosugar 1 and selenosugar 3 levels were clearly higher in the postsupplementation phase compared to the pre-supplementation phase (Fig. 4A and B). The elevation of the levels of these metabolites in the post-supplementation phase most likely results from increased loads of hydrogen selenide resulting from the action of selenocysteine lyase on the excess selenocysteine originating from the catabolism of the replenished selenoproteins following supplementation.
Selenosugar $\mathbf{3}$ is the deacetylated form of selenosugar $\mathbf{1}$, and is probably produced by a yet to be identified deacetylase enzyme. Jäger et al. has reported saturation trends for the production of selenosugar 3 under conditions of no supplementation. ${ }^{13}$ This trend can also be noticed in the present data as indicated by the initial sharp increase of selenosugar 3 with increased selenosugar 1 that becomes less pronounced at higher selenosugar 1 levels (Fig. 5D, G and H). The stepwise supplementation allowed us to further investigate this saturation trend. We expected, based on the observations of Jäger et al., ${ }^{13}$ that the percentage of selenosugar 3 of the total urinary selenium would decrease upon increasing Se supplementation. In contrast to this expectation, our supplementation regimen revealed that the production of selenosugar 3 actually continues to increase and seems to stabilize at $\sim 5 \%$ of total urinary selenium under high selenium intake (Fig. 5B).

\section{Se-methylselenoneine}

Se-methylselenoneine has recently been found to be a minor constituent of fish muscle, ${ }^{23}$ and is thought to result from the methylation of selenoneine, the selenium analogue of the antioxidant ergothioneine and a common major constituent of fish. ${ }^{24}$ Se-methylselenoneine in human urine was first reported by Klein et al., ${ }^{25}$ and it was found in all of the urine samples of our volunteers. The present study is the first to monitor the excretion of Se-methylselenoneine in response to selenium supplementation. The levels of Se-methylselenoneine did not 

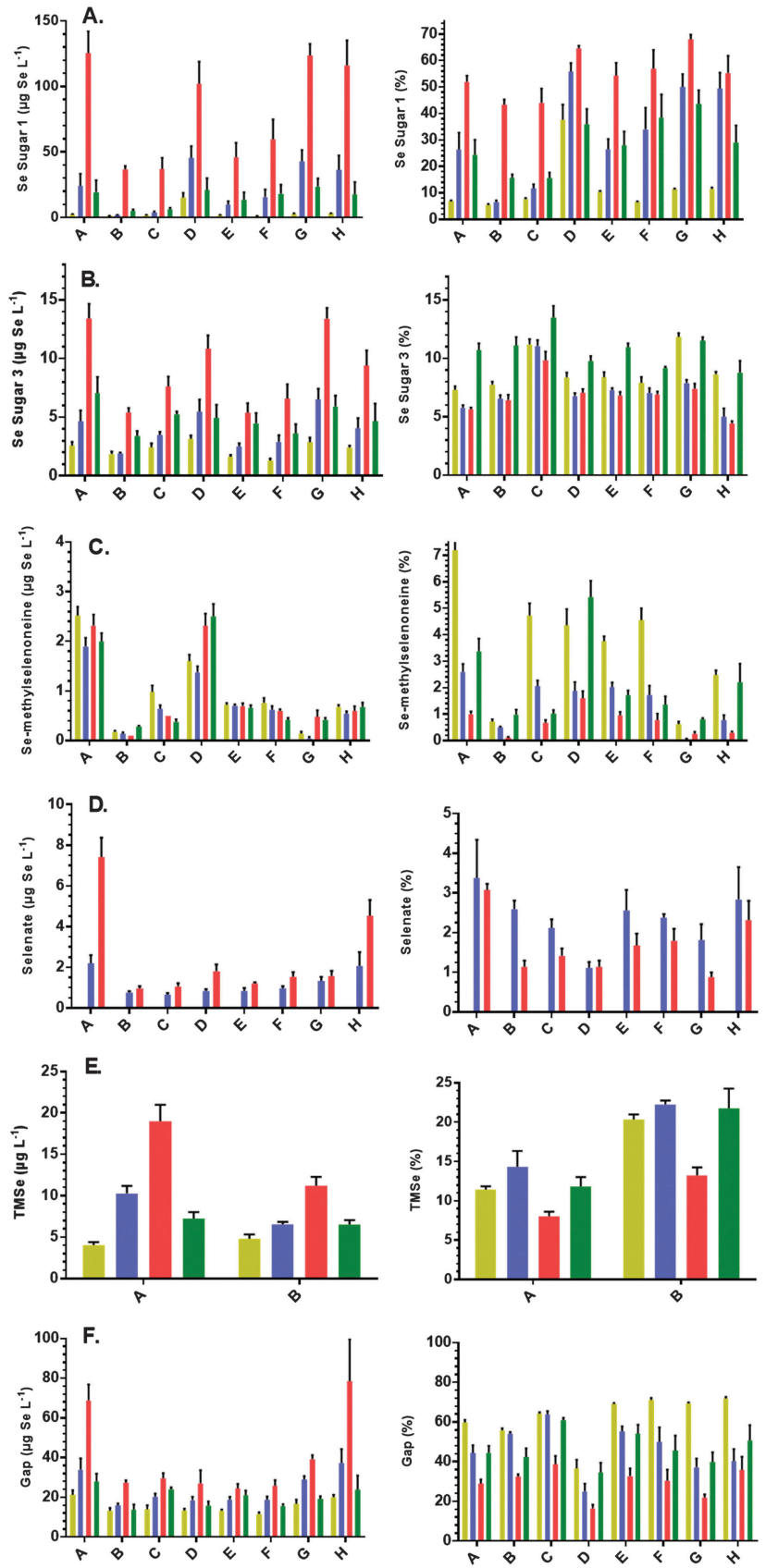

Fig. 4 The change in the levels of the selenium metabolites over the study phases shown with the normalized concentration (left) and percentage (right). Selenosugar 1 (A), selenosugar $\mathbf{3}$ (B), Se-methylselenoneine (C), selenate (D), trimethylselenonium (E), the gap between total urinary selenium and the sum of all analyzed species (F). The study phases are highlighted by different colors (see Fig. 2). Each bar shows the average for the five days of the respective phase with the standard error of the mean. Selenate was detected in all pre- and post-supplementation samples, but only at trace levels $\left(0.1-0.3 \mu \mathrm{g} \mathrm{Se} \mathrm{L}^{-1}\right)$ at around the limit of quantification $\left(0.2 \mu \mathrm{g} \mathrm{Se}^{-1}\right)$ for this selenium species (not displayed in Fig. 3D). Only two volunteers were TMSe producers ( $\mathrm{A}$ and $\mathrm{B}$ ).

correlate with selenosugar 1 levels (Fig. 5E), nor was the production of Se-methylselenoneine affected by selenite supplementation (Fig. 4C), suggesting that this metabolite is probably not in equilibrium with selenium body pools.

\section{Selenite and selenate}

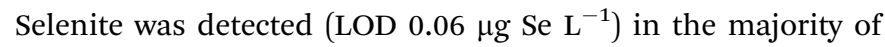
urine samples but it was always below the limit of quantification $\left(0.2 \mu \mathrm{g} \mathrm{Se} \mathrm{L}^{-1}\right)$, and did not show an increase in the supplementation phases. On the other hand, selenate was consistently detected in the pre-supplementation and postsupplementation phases in all volunteers with low levels scattered around the limit of quantification $\left(0.2 \mu \mathrm{g} \mathrm{Se} \mathrm{L}^{-1}\right)$. We found a consistent and marked increase in selenate excretion following selenite supplementation (Fig. 4D and Table 1), supporting a recent report showing the oxidation of administered selenite to selenate in humans. ${ }^{26}$ This oxidation reaction is possibly undertaken by the sulfite oxidase enzyme, in a manner similar to the oxidation of sulfite to the primary sulfur urinary metabolite sulfate. $^{27}$

A possible source of selenate in human urine without selenite supplementation is the diet, although selenate is generally of low abundance in food relative to selenoamino acids. ${ }^{28}$ Another possibility is the oxidation of hydrogen selenide in enzymatic pathways probably similar to that of hydrogen sulfide. ${ }^{29}$ Investigation of the post-supplementation levels of selenate provided some insight into the latter possibility. As discussed above, the selenosugars showed increased production even after the cessation of supplementation, which probably reflects high hydrogen selenide load due to replenished selenium body pools. In contrast to the selenosugar response, the levels of selenate showed an abrupt return to background values upon cessation of selenium supplementation (Fig. 4D and Table 1). Such a rapid response indicates that selenate production starts from the oxidation of the administered selenite rather than from the oxidation of hydrogen selenide (Fig. 1).

\section{Trimethylselenonium (TMSe)}

The genetically determined metabolite trimethylselenonium ${ }^{30}$ was produced in significant amounts by only 2 of our 8 volunteers (A and B, "TMSe producers", Fig. 4E). TMSe was detected in some of the urine samples of the other volunteers but always fell below

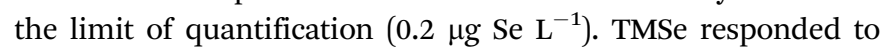
selenite supplementation but this response was less than that shown by selenosugar 1 which is in agreement with a previous report. ${ }^{31}$ In contrast to selenosugar 3 , the percentage of the total urinary selenium for TMSe was higher in the $200 \mu \mathrm{g}$ supplementation phase relative to the pre-supplementation phase (Fig. 4B and E). The urinary levels of TMSe in the two TMSe producers were also clearly higher than those of selenosugar 3 throughout all study phases (compare Fig. 4B and E). Therefore, TMSe comes after selenosugar 1 in the order of responsive selenometabolites to selenium intake.

The relatively small response of TMSe relative to selenosugar $\mathbf{1}$, and the clear dominance of selenosugar 1 in the $400 \mu \mathrm{g}$ supplementation phase, indicates that TMSe might not be a significant detoxification product of excess selenium, but rather a by-product of methylation enzymatic activity. This metabolite might lead to over-excretion and thus affect the selenium status and body pools. Indeed, a recent study showed that TMSe 
A.

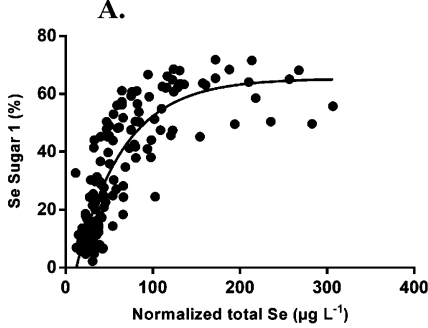

D.
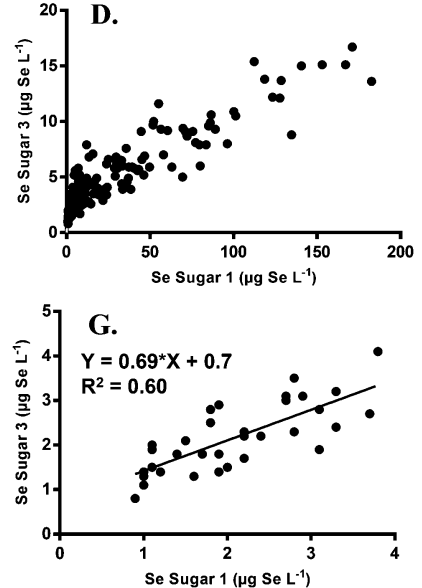
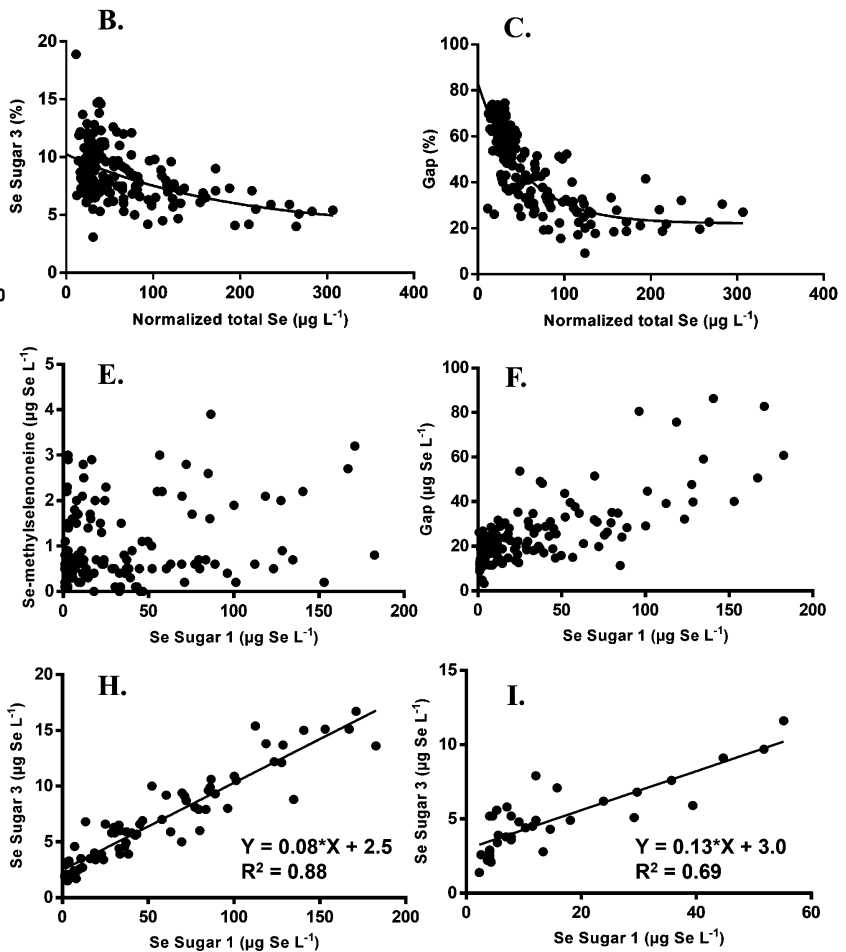

Fig. 5 The correlation between total urinary selenium and the percentage of selenosugar $\mathbf{1}$ (A), selenosugar $\mathbf{3}$ (B), and the gap (C) between total urinary selenium and the sum of all analyzed species, and the correlation between selenosugar 1 and selenosugar $\mathbf{3}$ (D), Se-methylselenoneine (E), and the gap (F) between total urinary selenium and the sum of all analyzed species. Graphs G-I are segments of graph D corresponding to the pre-supplementation, $200 \mu \mathrm{g}$ and $400 \mu \mathrm{g}$ Se supplementation, and post-supplementation phases, respectively. All concentrations were normalized according to specific gravity. Note the large decrease in slope from $\mathrm{G}$ to $\mathrm{H}$ which suggests saturation for the production of selenosugar $\mathbf{3}$ at high selenium intake, in agreement with the finding of Jäger et al. under conditions of no supplementation. ${ }^{13}$

producers had larger amounts of total selenium in urine and lower erythrocyte selenium to urinary selenium ratios compared to non-producers, suggesting that TMSe is excreted "on top" of the selenosugars. ${ }^{30}$ The necessity to investigate the correlation between TMSe production and selenoprotein expression, which is a more reliable biomarker for selenium status, has been discussed. ${ }^{30}$

\section{The missing selenium (the "selenium gap")}

In agreement with previous studies [e.g. ref. 12-14], the present study showed a large gap (Fig. $4 \mathrm{~F}$ ) between total urinary selenium and the sum of all selenium species measured by HPLC/ICPMS. Two explanations already discussed ${ }^{12}$ for the selenium gap are that it comprises many minor selenium species, the individual concentrations of which fall below the limit of detection, or that it represents selenium compounds that do not elute from the chromatographic columns.

The selenium gap clearly responded to supplementation (Fig. 4F) and correlated with selenosugar 1 production (Fig. 5F). With increased selenium intake, the contribution of selenosugar 1 to the total urinary selenium increases while that of the selenium gap decreases (Fig. 5A and C). However, at high selenium intake the dominance of selenosugar 1 ceases to increase and the contribution of the selenium gap and selenosugar 1 to the total urinary selenium starts to stabilize at $\sim 20 \%$ and $\sim 70 \%$, respectively (Fig. 5A and C). This trend can be explained by the presence of currently unknown selenium metabolites responsive to selenium administration that become more important at high selenium intake or upon saturation of the production of selenosugar 1.

To shed light on this finding, we also examined data from a previously published study involving the collection of 3-4 urine samples from 5 patients receiving extremely high dosage of selenomethionine $\left(4000 \mu \mathrm{g}\right.$ Se) as part of their chemotherapy, ${ }^{31}$ and calculated the selenium gap based on the difference between the reported values for total urinary selenium and the individual species. Our calculations indicated that the above-mentioned trends seem to extend to these extremely high selenium intake levels (average selenosugar 1 percentage $76 \%$ vs. $13 \%$ for the selenium gap). It should be borne in mind, however, that the selenium form administered in that study was selenomethionine rather than selenite, and that the volunteers were critically ill patients and therefore may not reflect normal physiological conditions.

Future work will investigate further this "selenium gap" with the goal of identifying its components.

\section{References}

1 F. Zinoni, A. Birkmann, W. Leinfelder and A. Bock, Cotranslational insertion of selenocysteine into formate dehydrogenase from Escherichia coli directed by a UGA codon, Proc. Natl. Acad. Sci. U. S. A., 1987, 84, 3156-3160. 
2 M. V. Kasaikina, D. L. Hatfield and V. N. Gladyshev, Understanding selenoprotein function and regulation through the use of rodent models, Biochim. Biophys. Acta, Mol. Cell Res., 2012, 1823, 1633-1642.

3 K. Schwarz and C. M. Foltz, Selenium as an Integral Part of Factor-3 against Dietary Necrotic Liver Degeneration, J. Am. Chem. Soc., 1957, 79, 3292-3293.

4 N. Esaki, T. Nakamura, H. Tanaka, T. Suzuki, Y. Morino and K. Soda, Enzymatic synthesis of selenocysteine in rat liver, Biochemistry, 1981, 20, 4492-4496.

5 R. F. Burk, K. E. Hill and A. K. Motley, Plasma selenium in specific and non-specific forms, Biofactors, 2001, 14, 107-114.

6 N. Esaki, T. Nakamura, H. Tanaka and K. Soda, Selenocysteine lyase, a novel enzyme that specifically acts on selenocysteine. Mammalian distribution and purification and properties of pig liver enzyme, J. Biol. Chem., 1982, 257, 4386-4391.

7 H. E. Ganther, Reduction of the selenotrisulfide derivative of glutathione to a persulfide analog by glutathione reductase, Biochemistry, 1971, 10, 4089-4098.

8 J. Lu, C. Berndt and A. Holmgren, Metabolism of selenium compounds catalyzed by the mammalian selenoprotein thioredoxin reductase, Biochim. Biophys. Acta, 2009, 1790, 1513-1519.

9 M. Bjornstedt, S. Kumar and A. Holmgren, Selenite and selenodiglutathione: reactions with thioredoxin systems, Methods Enzymol., 1995, 252, 209-219.

10 S. C. Low, J. W. Harney and M. J. Berry, Cloning and functional characterization of human selenophosphate synthetase, an essential component of selenoprotein synthesis, J. Biol. Chem., 1995, 270, 21659-21664.

11 Y. Kobayashi, Y. Ogra, K. Ishiwata, H. Takayama, N. Aimi and K. T. Suzuki, Selenosugars are key and urinary metabolites for selenium excretion within the required to low-toxic range, Proc. Natl. Acad. Sci. U. S. A., 2002, 99, 15932-15936.

12 D. Kuehnelt, N. Kienzl, P. Traar, N. H. Le, K. A. Francesconi and T. Ochi, Selenium metabolites in human urine after ingestion of selenite, L-selenomethionine, or DL-selenomethionine: a quantitative case study by HPLC/ICPMS, Anal. Bioanal. Chem., 2005, 383, 235-246.

13 T. Jäger, H. Drexler and T. Goen, Ion pairing and ion exchange chromatography coupled to ICP-MS to determine selenium species in human urine, J. Anal. At. Spectrom., 2013, 28, 1402-1409.

14 T. Jäger, H. Drexler and T. Goen, Human metabolism and renal excretion of selenium compounds after oral ingestion of sodium selenite and selenized yeast dependent on the trimethylselenium ion (TMSe) status, Arch. Toxicol., 2016, 90, 1069-1080.

15 B. Gammelgaard, S. Sturup and M. V. Christensen, Human urinary excretion and metabolism of (82)Se-enriched selenite and selenate determined by LC-ICP-MS, Metallomics, 2012, 4, 149-155.
16 S. Kokarnig, A. Tsirigotaki, T. Wiesenhofer, V. Lackner, K. A. Francesconi, S. A. Pergantis and D. Kuehnelt, Concurrent quantitative HPLC-mass spectrometry profiling of small selenium species in human serum and urine after ingestion of selenium supplements, J. Trace Elem. Med. Biol., 2015, 29, 83-90.

17 D. Kuehnelt, D. Juresa, N. Kienzl and K. A. Francesconi, Marked individual variability in the levels of trimethylselenonium ion in human urine determined by HPLC/ ICPMS and HPLC/vapor generation/ICPMS, Anal. Bioanal. Chem., 2006, 386, 2207-2212.

18 E. H. Larsen and S. Sturup, Carbon-enhanced inductively coupled plasma mass spectrometric detection of arsenic and selenium and its application to arsenic speciation, J. Anal. At. Spectrom., 1994, 9, 1099-1105.

19 L. Levine and J. P. Fahy, Evaluation of urinary lead determinations, J. Ind. Hyg. Toxicol., 1945, 27, 217-223.

20 D. Juresa, J. Darrouzes, N. Kienzl, M. Bueno, F. Pannier, M. Potin-Gautier, K. A. Francesconi and D. Kuehnelt, An HPLC/ICPMS study of the stability of selenosugars in human urine: implications for quantification, sample handling, and storage, J. Anal. At. Spectrom., 2006, 21, 684-690.

$21 \mathrm{~J}$. L. Hoffman, Ion chromatographic analysis of the purity and synthesis of sulfonium and selenonium ions, J. Chromatogr., 1991, 588, 211-216.

22 P. Traar, F. Belaj and K. A. Francesconi, Synthesis of Methyl 2-Acetamido-2-deoxy-1-seleno- $\beta$-D-gluco- and galacto-pyranoside: Selenium Metabolites in Human Urine, Aust. J. Chem., 2004, 57, 1051-1053.

23 N. Kroepfl, K. B. Jensen, K. A. Francesconi and D. Kuehnelt, Human excretory products of selenium are natural constituents of marine fish muscle, Anal. Bioanal. Chem., 2015, 407, 7713-7719.

24 Y. Yamashita and M. Yamashita, Identification of a novel selenium-containing compound, selenoneine, as the predominant chemical form of organic selenium in the blood of bluefin tuna, J. Biol. Chem., 2010, 285, 18134-18138.

25 M. Klein, L. Ouerdane, M. Bueno and F. Pannier, Identification in human urine and blood of a novel selenium metabolite, Se-methylselenoneine, a potential biomarker of metabolization in mammals of the naturally occurring selenoneine, by HPLC coupled to electrospray hybrid linear ion trap-orbital ion trap MS, Metallomics, 2011, 3, 513-520.

26 T. Jäger, H. Drexler and T. Goen, Human metabolism and renal excretion of selenium compounds after oral ingestion of sodium selenate dependent on trimethylselenium ion (TMSe) status, Arch. Toxicol., 2016, 90, 149-158.

27 H. J. Cohen and I. Fridovich, Hepatic sulfite oxidase. The nature and function of the HEME prosthetic groups, J. Biol. Chem., 1971, 246, 367-373.

28 P. D. Whanger, Selenocompounds in plants and animals and their biological significance, J. Am. Coll. Nutr., 2002, 21, 223-232.

29 T. M. Hildebrandt and M. K. Grieshaber, Three enzymatic activities catalyze the oxidation of sulfide to thiosulfate in 
mammalian and invertebrate mitochondria, FEBS J., 2008, 275, 3352-3361.

30 D. Kuehnelt, K. Engstrom, H. Skroder, S. Kokarnig, C. Schlebusch, M. Kippler, A. Alhamdow, B. Nermell, K. Francesconi, K. Broberg and M. Vahter, Selenium metabolism to the trimethylselenonium ion (TMSe) varies markedly because of polymorphisms in the indolethylamine $N$-methyltransferase gene, Am. J. Clin. Nutr., 2015, 102, 1406-1415.

31 D. Kuehnelt, D. Juresa, K. A. Francesconi, M. Fakih and M. E. Reid, Selenium metabolites in urine of cancer patients receiving L-selenomethionine at high doses, Toxicol. Appl. Pharmacol., 2007, 220, 211-215. 